\title{
The retrograde approach to coronary chronic total occlusion interventions
}

\author{
I. Ungi, P. Tajti, P. Hausinger, A. Katona, V. Sasi \& A. Thury \\ Department of Cardiology, University of Szeged, Szeged, Hungary
}

Keywords

chronic total occlusion, percutaneous

coronary intervention, retrograde approach

Correspondence
I. Ungi, Department of Cardiology, University
of Szeged, Szeged, Hungary.
Tel: +3662546497; Fax: +3662342616;
E-mail: ungi.imre@gmail.com

Funding Information

No funding information provided.

Continuing Cardiology Education, 2015; 1(1), doi: $10.1002 /$ cce2.3

\begin{abstract}
The results of percutaneous coronary interventions (PCI) dramatically improved in the last decades. Despite this improvement chronic total coronary occlusions (CTO) still remain a major challenge of interventional cardiology due to the complexity of these lesions. Retrograde recanalization proved to be one of the most significant amendments of the technique. Careful assessment of the occluded vessel, the donor artery and the interventional collaterals has fundamental importance for a safe and efficient procedure. High-quality diagnostic angiogram and other imaging methods also have paramount importance for the success. The proper selection of the vascular access for retrograde CTO PCI can be challenging in patients with peripheral artery disease because of special requirements toward the guiding catheters. The latest publications demonstrated continuous improvement in the results together with decreasing complication rates of retrograde procedures. This technique significantly increased the number of patients who can be treated with PCI even with failed previous antegrade attempt.
\end{abstract}

Answer questions and earn CME: https://www.wileyhealthlearning.com/ Activity2/3700518/Activity.aspx

\section{Introduction}

The contemporary techniques and devices of percutaneous coronary interventions (PCIs) significantly improved the results of treating coronary artery disease and allowed us to treat more and more complex lesions with reasonably low complication rate. Despite this development, the success rate of catheter-based revascularization in chronic total coronary occlusions (CTOs) still remains significantly lower compared to that of non-CTO lesions. Numerous devices and techniques have been developed to improve the results of the conventional antegrade approach, however, the majority of them have not proved to be beneficial compared to traditional guidewire crossing.

The retrograde route for balloon angioplasty was applied first in 1990 for the treatment of non-occlusive coronary artery disease [1]. The rationale of choosing the retrograde route was established by histopathologic studies that demonstrated communications between lumen recanalization channels and intraplaque capillaries [2]. Ozawa proposed that these channels have a branched tree-like configuration that spreads out from the proximal toward the distal cap (Figure 1) and this configuration may lead the antegrade wire into the subintimal space [3]. The other reason why the entry into the body of CTO is easier from the distal vascular bed is that the proximal cap is getting harder and thicker with aging of the occlusion [4] while the distal cap remains softer and is typically tapered [3]. Surmely et al. published first in 2007 retrograde CTO recanalization in 21 patients via septal collaterals, and since then still this route is the most frequently used approach for retrograde CTO PCI [5].

\section{Case Selection for Retrograde Recanalization}

The antegrade route should generally be considered as the primary approach; however, prior to all the CTO procedures it is essential to assess the eligibility of the patient and the lesion for a retrograde approach in order to be prepared to change the strategy. Retrograde recanalization is uncommon as a first choice but there are a few scenarios and types of coronary pathology that are completely unsuitable for an 


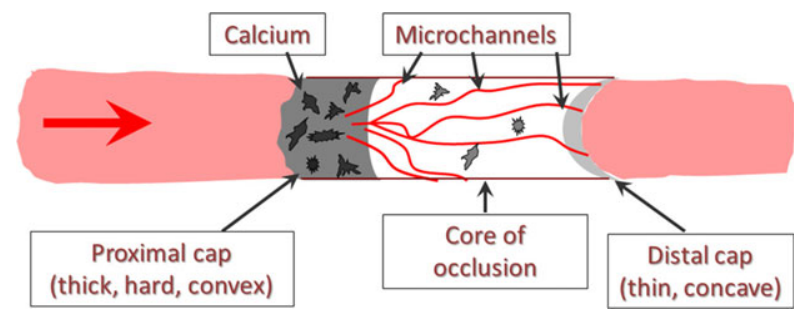

Figure 1. Structure of the chronic total occlusions of advanced age. Typical characteristics of these lesions are convexity and thickness of the proximal and concavity of the distal cap, diverging course of the microchannels. The lesions with these features are easier to cross in the retrograde direction than in the antegrade direction.

antegrade approach: (1) aorto-ostial occlusions of the right coronary artery (RCA); (2) previously attempted and failed antegrade recanalization by an experienced operator; (3) very unfavorable morphology for antegrade strategy (seriously diseased proximal segment with cap ambiguosity) and favorable anatomy for the retrograde (straightforward collateral pathway, large distal vessel, easy distal cap).

Initially, retrograde CTO PCI had been considered as a competitor of the antegrade PCI techniques; recently, however, the widespread adoption of the "hybrid" approach has changed this view. Following the hybrid strategy the operator should alternate dynamically between the diverse recanalization methods [6]. Discussion of the hybrid strategy would go beyond the scope of this paper but a schematic algorithm of the hybrid approach prepared by Brilakis et al. [6] is presented in Figure 2. This flowchart makes the place of retrograde CTO recanalization easy to understand in the contemporary CTO PCI practice. In further sections of this article we primarily focus on the specific clinical and technical aspects of the retrograde route.

\section{Options for the retrograde route}

\section{Septal collaterals}

It was discovered earlier that the route through septal collaterals between the left anterior descending artery and the RCA are reasonably safe to cross, and even balloon dilation can be performed in these channels without a considerable risk [5]. First, Levin described the anatomy of collateral pathways and found that the majority of the connections between the left and right coronary systems were septal (42\%) while the atrial epicardial (32\%), interatrial (18\%) and bridging $(8 \%)$ proved to be significantly less frequent [7]. The advantages of septal collaterals are that (1) they usually are multiple with a wide range of selection; (2) they usually are straighter than the epicardial vessels; (3) an accidental injury will not result in pericardial tamponade, and the septal hematoma is usually benign (Figure 3). However, in some cases, before joining the RCA, there is a short epicardial course, that is, the risk of pericardial tamponade cannot be fully excluded using the septal collaterals. The size of the branch is one of the most important predictors of successful passage with microcatheters. A semiquantitative classification of the development of collaterals was described by Werner; this classification is generally used for strategy planning [10].

\section{Epicardial collaterals}

Epicardial channels are larger in size hence they seem to be more suitable for the retrograde access. But they usually have much more tortuosity than septals; consequently, there is a significant risk of vessel injury and

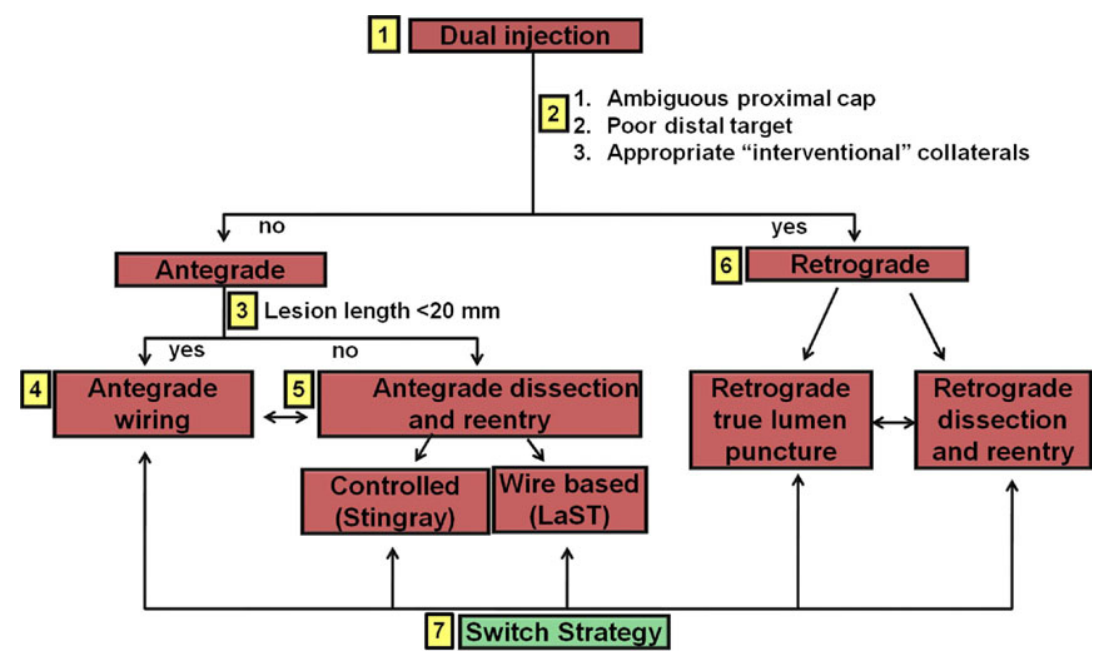

Figure 2. Algorithm of chronic total occlusion strategy by the "Hybrid" approach. Reprinted from JACC: Cardiovascular Interventions, 5, S Emmanouil, J Brilakis, JA Grantham et al. A Percutaneous Treatment Algorithm for Crossing Coronary Chronic Total Occlusions, p367-379, Copyright (2012), with permission from American College of Cardiology Foundation. 


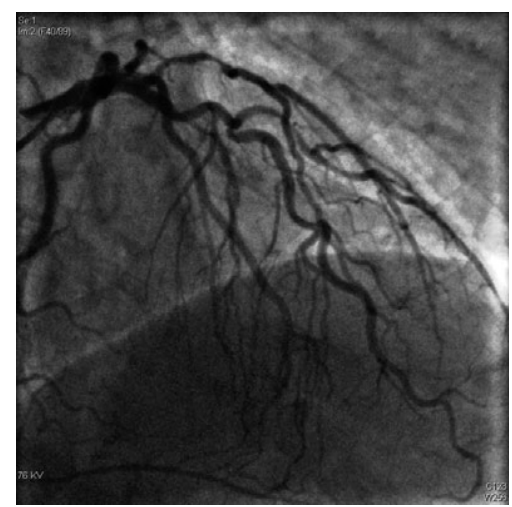

Figure 3. Septal collaterals (Grade Werner 2) from the LAD to the PD branch of the RCA. Note that the channels are relatively straight and there is a clear connection with the recipient vessel. LAD, left anterior descending; PD, posterior descending; RCA, right coronary artery.

pericardial effusion (Figure 4). Therefore, pericardial collaterals are only used as secondary options in retrograde routes. The threshold for choosing epicardial vessels is significantly lower in post-bypass patients [11], because the post-operative scar lowers the risk of pericardial tamponade, however, it does not prevent it completely [12].

The ideal collateral channel for the retrograde access of the distal cap should have the following characteristics:

possibly septal collaterals;

visible direct connection with the recipient vessel (at least Werner 1 grade);

relatively straight or have shallow bends only;

angles with both the donor and recipient vessels are $<90^{\circ}$;

entry of the collateral into the recipient artery is not close to the distal cap.

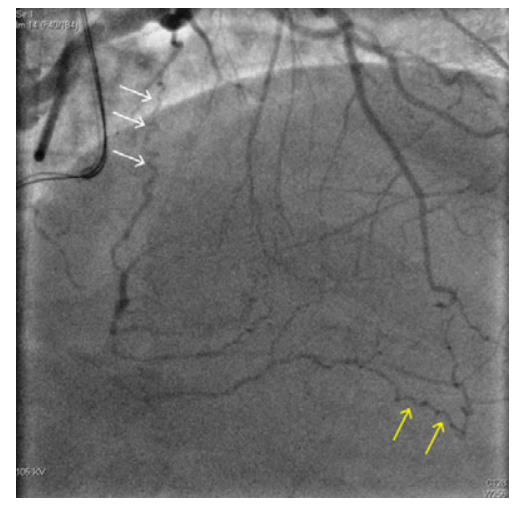

Figure 4. Tortuous epicardial collaterals from the LCX to the right PL branch (white arrows) and from the apical segment of the LAD to the marginal branch (yellow arrows). LCX, left circumflex artery; PL, posterolateral; LAD, left anterior descending.

\section{Optimal views for collateral tracking}

A single view can be misleading for the precise assessment of the channel course; usually two orthogonal projections are necessary. It is advisable to set some standard views during the diagnostic angiography (Table 1) that are extremely useful for planning of the retrograde strategy.

\section{Angiographic assessment of the occluded vessel}

Beyond the collaterals, the proper assessment of the occluded vessel is indispensable for the success of the retrograde procedure. The main points of this analysis are (1) assessment of the proximal segment (ostial stenosis, proximal disease, tortuosity, etc.) influencing the antegrade component and the retrograde passage; (2) morphology of the proximal cap (V-like or blunt, ambiguity, side branch, etc.); (3) occluded segment (length, calcification, bending, sidebranch at the distal cap); (4) quality of the distal vessel (diameter, calcification, serial occlusions, diffuse disease). All characteristics of the occluded artery, the donor vessel and collaterals must be taken into consideration for a proper planning of the interventional strategy [13].

\section{Vascular Access}

Almost all complex CTO procedures require double vascular access, however, retrograde recanalization somewhat differs from the antegrade procedure, when the visualization of the distal vascular bed is the only goal. In antegrade-only procedures the support and the diameter of the catheter in the contralateral artery have less importance, while for retrograde PCI a stable guiding position and strong support are required. This usually needs a 7F guiding, and because the antegrade catheter must usually

Table 1. Typical views for the assessment of the collateral supply of each of the coronary segments.

\begin{tabular}{lll}
\hline Collateral & Angulation & Note \\
\hline Septal & RAO cran & Origin from LAD \\
& RAO caud & Junction to PD \\
Epicardial (RV channel) & RAO cran & LCX-RCA, diagonal-LCX \\
& AP cran & Junction to LAD \\
PL channel & RAO cran & LCX-RCA \\
Atrial & LAO & Channels in anterolateral wall \\
& LAO cran & \\
& RAO cran & \\
& AP caud & Origin of the donor vessel \\
\hline
\end{tabular}

$\mathrm{RAO}$, right anterior oblique; $\mathrm{LAD}$, left anterior descending; $\mathrm{PD}$, posterior descending; LCX, left circumflex artery; RCA, right coronary artery; $\mathrm{PL}$, posterolateral; $\mathrm{RV}$, right ventricular; $\mathrm{AP}$ : antero-posterior; LAO: left anterior oblique. 
be 7 or $8 \mathrm{~F}$, as well, the most frequently applied strategy is bifemoral. Nevertheless, also the radial artery can be used if its size is compatible with a $7 \mathrm{~F}$ or with a $6 \mathrm{~F}$ catheter when an easy collateral passage is predicted. Some operators apply a "hybrid" vascular access with one femoral and one radial access in order to decrease the risk of vascular complications while keeping the possibility of using a large diameter catheter for the antegrade route. There are some operators who are experienced in both transradial access and CTO PCI, preferring a fully radial approach for the retrograde techniques with comparable results to that of a bifemoral access $[8,9]$, but this strategy definitely has limitations in complex cases when the use of multiple bulky devices can be necessary.

Using femoral access in diffusely atherosclerotic patients, the descending aorta and iliac arteries may have severe tortuosity that interferes with maneuverability of the wires and catheters. This difficulty can be eliminated with the routine use of long $(23$ or $45 \mathrm{~cm}$ ) kink-resistant sheaths. A specific requirement for the guiding catheters in retrograde procedures is that in the donor vessel a short guiding is advisable because the long route through a distal collateral can make the crossing of the lesion impossible with a $150 \mathrm{~cm}$ long microcatheter.

\section{Technical Details of the Procedure}

\section{Collateral channel navigation}

The collateral channel can be accessed with a workhorse wire; then, it can be changed through the microcatheter to a dedicated guidewire. For tracking the collaterals, a hydrophilic non-tapered wire is used; currently Sion, Fielder FC (Asahi Intecc Co., Ltd., Nagoya-shi, Japan), or Fielder XT-R (Asahi Intecc Co., Ltd.) are the most appropriate wires with a $1 \mathrm{~mm}$ long $45-90^{\circ}$ angle tip. The manipulation must be gentle in order to avoid collateral injury or perforation. Overlapping branches often cause confusion in following the track of the optimal channel. This problem can be solved by repeated selective tip injection through the microcatheter. Wiring of the septal collaterals is relatively safe, while crossing the tortuous epicardial collaterals requires particular care to avoid perforation and pericardial tamponade. Once the devices are positioned in the collateral, the antegrade flow is usually blocked; in these cases the wire manipulation is done blindly. Repeated tip injections may facilitate a safe and effective crossing of the epicardial channel. When the wire has crossed the collateral pathway, the microcatheter can be advanced to the distal cap of the occlusion. If the microcatheter cannot pass the collateral along the wire, it can be changed to a small diameter monorail balloon catheter. Serial low-pressure dilatations over the course of the collateral eliminate this difficulty in most cases. This maneuver, however, can be applied in septal channels only, because a possible side effect is the rupture of the collateral. In some cases, a temporary increase in the guiding support by deep cannulation or by anchoring technique can facilitate the tracking of the microcatheter.

\section{Crossing the occlusion}

The most common reason to start retrograde access to the occlusion is when the antegrade wiring has failed. The antegrade wire is left in the subintimal space and used later as a marker or for reverse controlled antegrade retrograde subintimal tracking (CART).

There are various techniques for crossing the occlusion with the retrograde wire. The fastest way is when a retrograde wire is directly passed into the proximal true lumen; however, this is only successful in the minority of cases, particularly with the floppy wire used for crossing the collateral (Figure 5A). With the wire escalation technique, this first wire is exchanged for stiffer and stiffer ones. But the retrograde wire escalation technique may fail in a considerable number of patients depending on the morphological characteristics of the lesion.

The kissing wire technique is applied for facilitation of antegrade wiring of the distal true lumen, in which the retrograde wire is used as a guide for manipulations with the antegrade one (Figure 5B).

These two "true lumen wiring techniques" were successful in more than half of the patients as presented in Table 2, although the distribution of the successful crossing techniques has a wide variation among the different patient cohorts.

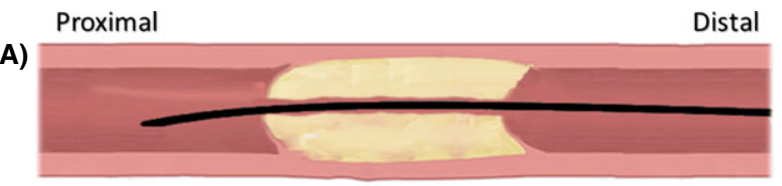

(B)

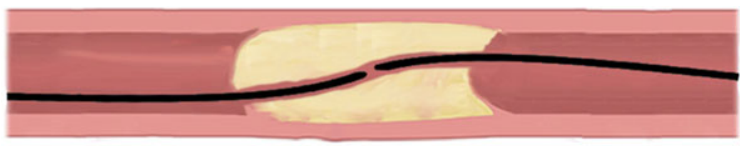

(C)

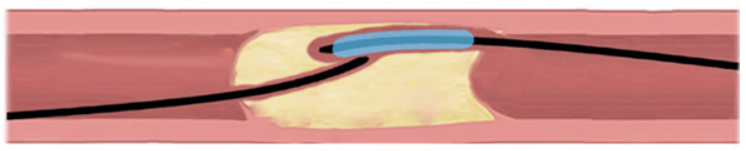

(D)

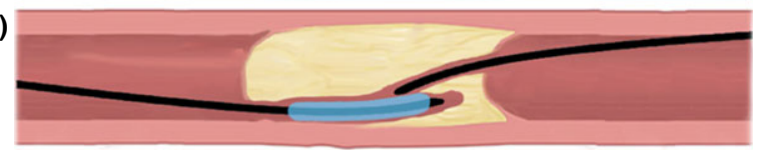

Figure 5. Schematic illustration of the retrograde crossing techniques. (A) Direct retrograde crossing; (B) Kissing wires; (C) Classical controlled antegrade retrograde subintimal tracking (CART); (D) Reverse CART. 
Table 2. Distribution of each retrograde crossing technique as a percentage of the successful retrograde recanalizations.

\begin{tabular}{|c|c|c|c|c|c|c|}
\hline Source (year) & $\begin{array}{l}\text { Number of successful } \\
\text { retrograde }\end{array}$ & $\begin{array}{l}\text { Direct wire } \\
\text { cross }\end{array}$ & $\begin{array}{l}\text { Kissing } \\
\text { wire }\end{array}$ & CART & $\begin{array}{l}\text { Reverse } \\
\text { CART }\end{array}$ & Reference \\
\hline CART Registry (2009) & 179 & 30 & 7 & 55 & 8 & {$[16]$} \\
\hline Korean Single Center (2010) & 18 & 44 & 22 & 22 & 11 & [15] \\
\hline Euro CTO Registry (2011) & 151 & 37 & 22 & $32^{1}$ & & [19] \\
\hline Belgrade Single Center (2012) & 36 & 31 & 25 & 17 & 22 & [18] \\
\hline US Multicenter Retrograde Registry (2012) & 376 & $43^{2}$ & & 11 & 46 & {$[20]$} \\
\hline $\begin{array}{l}\text { Japanese Retrograde } \\
\text { Summit Group (2013) }\end{array}$ & 266 & 23 & 23 & 12 & 42 & [14] \\
\hline $\begin{array}{l}\text { Multicenter Single-Operator Registry } \\
\text { (2013) }\end{array}$ & 228 & 27 & 54 & 10 & 9 & {$[17]$} \\
\hline
\end{tabular}

CART, controlled antegrade retrograde subintimal tracking; CTO, chronic total occlusion.

${ }^{1}$ CART and reverse CART are not given separately.

${ }^{2}$ Combined result of direct antegrade and retrograde crossing and kissing wire technique.

A major difficulty in direct retrograde wire crossing is poor wire control due to the long route through the donor artery, the collateral channel and the distal vessel. This may be eliminated at least partially by using a supportive microcatheter, like Corsair (Asahi Intecc Co., Ltd., 1703 Wakita-cho, Moriyama-ku, Nagoya-shi, Aichi 463-0024 Japan), and the retrograde wire escalation technique. In case of failed true lumen recanalizations more complex techniques such as the CART technique or the reverse CART technique are attempted (Figures 5C and D). The essence of the CART technique is that in the case of an inadvertent subintimal position of the antegrade wire a connection is created between the subintimal space and the distal true lumen by retrograde balloon dilation at the distal cap [21]. This maneuver facilitates getting the antegrade wire into the distal true lumen. The availability of the Corsair catheter with its unique wire support decreased the need for the CART technique, because it considerably improves the progress with the retrograde wire. With the reverse CART technique, the connection is made between the subintimal space and the proximal true lumen by antegrade ballooning at the level of the proximal cap, resulting in a passage of the retrograde wire into the proximal true lumen. In case of a long and hard lesion and/or unclear vessel course, the knuckle wire technique can be the key to the solution. With the small loop shaped at the tip of the wire, a subintimal space can be created for the subsequent reverse CART technique. A frequently occurring difficulty in reverse CART is the immediate collapse of the lumen after deflating the balloon on the antegrade wire. Aiming the inflated reverse CART balloon with the retrograde wire tip may eliminate this problem, or introducing a GuideLiner Vascular Solutions Inc., 6464 Sycamore Court North Minneapolis, MN 55369 USA) catheter on the antegrade wire into the enlarged space may help in capturing and externalizing the retrograde wire [22]. In some cases, after failed balloon dilation, deployment of a stent can keep the connection open between the proximal true lumen and the subintimal space, referred to as the "reverse stent CART technique" [23].

\section{Intravascular ultrasound guided reverse CART}

Although reverse CART can usually be performed with angiographic guidance only, the optimal size of the balloon and/or the correct position of the wires, that is, intimal or subintimal, cannot be established with certainty in some cases. This difficulty leads to several failed attempts at finding the proximal true lumen with the retrograde wire. This results in retrograde dissection enlargement and multiple false lumen creation. This complication can be avoided by using intravascular ultrasound (IVUS) that provides information on (1) the exact position of the connection between the intima and subintima; (2) visual information on the distance of the retrograde wire to the intima; (3) the correct balloon size for medial disruption. If the lumen collapsed after dilation with a properly sized balloon, the above-mentioned devices (stent, GuideLiner) or temporary lumen scaffolding with a snare wire may keep the channel open while the true lumen is found with the retrograde wire (Figure 6).

\section{Steps after crossing the lesion}

In the case of retrograde crossing, there are two options to pre-dilate the lesion and finalize the procedure.

\section{Retrograde opening with antegrade rewiring of the lesion}

The tip of the retrograde wire can be directed toward the antegrade guiding catheter, and by simultaneous maneu- 


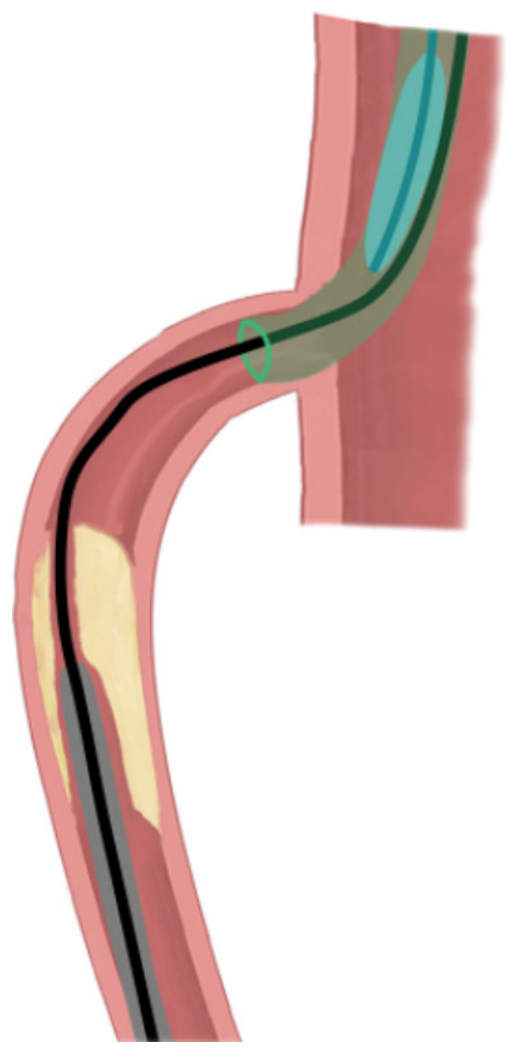

Figure 6. Schematic illustration of anchoring the retrograde guidewire in the antegrade guiding catheter with a trapping balloon.

vers with both the wire and the guiding, the wire can be introduced into the catheter. Initially, using the high profile microcatheters with limited crossability, they had to be exchanged for a low profile balloon and the lesion was dilated retrogradely. After that an antegrade wire could usually be introduced into the distal true lumen. In the case of successful antegrade wiring, the retrograde gear could be removed and the pre-dilation and stenting were completed on the antegrade wire. A significant drawback of this strategy was an inadvertent subintimal wiring due to the dissected vessel wall, which interfered with the successful recanalization.

\section{Externalization strategy}

The unfavorable outcome of the previous approach can be prevented by crossing the lesion with the retrograde microcatheter and introducing it into the guiding followed by externalization of the long wire through the antegrade side. This externalization strategy requires a microcatheter with excellent support, trackability, and crossability, characteristics that are available with the Corsair catheter. However, even with Corsair, the retrograde crossing needs anchoring of the retrograde wire in the guiding catheter with inflation of a trapping balloon (Figure 7). The wire used for crossing the lesion retrogradely then can be changed to a dedicated externalization wire (RG3), which is used in the remaining part of the procedure in the antegrade direction. The advent of the Corsair catheter and the RG3 wire has been a significant steps in the retrograde strategy, which made the results of this approach more predictable.

In some cases, introduction of the retrograde wire into the antegrade guiding catheter is not successful due to ostial CTO, seriously diseased proximal segment, or a very proximal re-entry into the true lumen. In these cases, the retrograde wire can be captured by a snare in the ascending aorta and the antegrade guiding catheter can be pulled gently into the ostium by the unit of the wire and the snare (Figure 8). This snare-wire technique can further improve the results of the retrograde approach.

The detailed description of the final phase of a retrograde CTO recanalization is beyond the scope of this article. This part of the procedure does not differ considerably from that of the antegrade procedures. An important point, however, is that the retrograde wire and the microcatheter must be removed with particular care after successful pre-dilation and stenting the lesion.

The externalized wire is used for the antegrade ballooning and stenting; therefore, the microcatheter must be pulled back into the collateral before that step, and after stenting, it must be advanced again to protect the vessel and collaterals from an injury during removal of the wire. Before removal of the retrograde gear, a contralateral angiogram is indispensable to check the donor artery before leaving the vessel without protection. After that, the externalized wire must be pulled back into the microcatheter and they should be withdrawn as a single unit in order to protect the collateral from a wire-induced injury again.

\section{Outcome and Complications of Retrograde CTO Recanalization}

\section{Procedural results}

The short- and long-term angiographic and clinical results have been presented by several CTO centers as those of single-center or multi-center registries. The outcome of the first small patient cohort was reported in 2008 by Biondi-Zoccai et al. with the conclusion that retrograde PCI is a feasible and safe alternative to the antegrade approach [24]. The first large series of unselected cases was reported in 2009 from the Toyohashi Heart Center [25]. Although the success rate proved to be moderate $(65.6 \%)$ in this population, $75.2 \%$ of these patients previously underwent a failed antegrade attempt, and the 


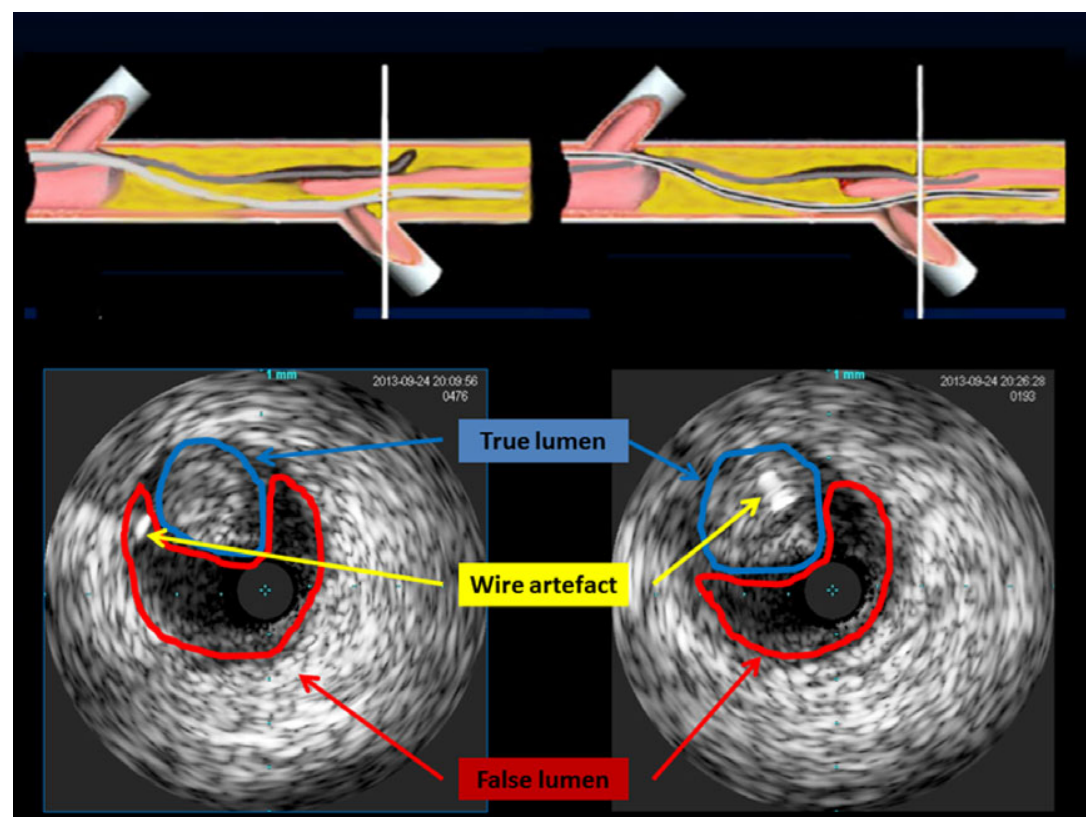

Figure 7. Localization of the guidewire by IVUS guidance. On the left side of the figure, both the guidewire and IVUS catheter are in the subintimal space, while on the right side, IVUS confirmed the entry of the wire into the true lumen.

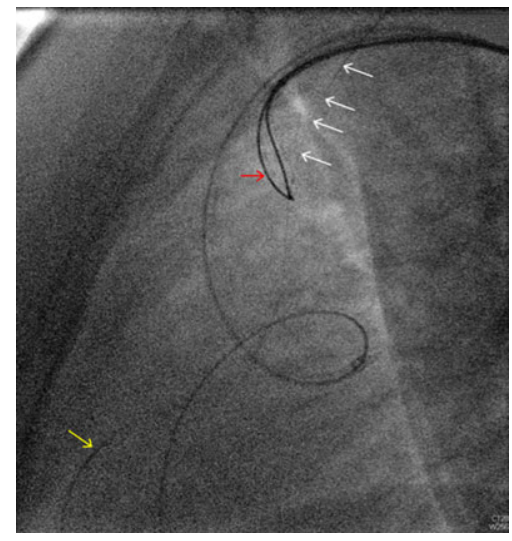

Figure 8. Capture of the retrograde guidewire for externalization during recanalization of a right coronary artery. White arrows: guidewire in the ascending aorta; red arrow: opened snare capturing the guidewire; yellow arrow: Corsair microcatheter.

overall success rate was $85 \%$ with a reasonably low complication rate and no mortality.

All relevant published data on the outcome of retrograde CTO PCI were collected, analyzed and reported this year by El Sabbagh et al. [26]. They performed a meta-analysis of 26 studies with the procedural and clinical data of 3482 patients undergoing a retrograde CTO PCI between 2006 and 2013. The overall procedural success rate proved to be $83.3 \%$ with a $74.5 \%$ retrograde success. Mortality remained as low as $0.7 \%$ and the major adverse cardiac event rate was also reasonably moderate at $2 \%$. The skill and selected strategy of the diverse centers must have been variable as indicated by a wide range of occurrence of certain complications, for

Table 3. Proportion of studies reporting success and various complication rates of retrograde CTO $\mathrm{PCl}$. Reprinted from International Journal of Cardiology, 2, A El Sabbagh, VG Patel, OM Jeroudi et al. Angiographic success and procedural complications in patients undergoing retrograde percutaneous coronary chronic total occlusion interventions: A weighted meta-analysis of 3482 patients from 26 studies, p243-248, Copyright (2014), with permission from Elsevier.

\begin{tabular}{lr}
\hline Variable & $\begin{array}{l}\text { Proportion of studies reporting } \\
\text { outcome }\end{array}$ \\
\hline Procedural success & $24 / 26(0.92)$ \\
Death & $22 / 26(0.84)$ \\
Emergent coronary artery bypass & $19 / 65(0.73)$ \\
graft surgery & \\
Stroke & $9 / 26(0.35)$ \\
Myocardial infarction & $15 / 26(0.58)$ \\
Q wave myocardial infarction & $12 / 26(0.46)$ \\
Coronary perforation (per lesion) & $8 / 26(0.31)$ \\
Collateral perforation & $21 / 26(0.81)$ \\
Tamponade & $18 / 26(0.69)$ \\
Vascular complication & $426(0.15)$ \\
Major bleed & $3 / 26(0.11)$ \\
Contrast nephropathy & $5 / 26(0.19)$ \\
Radiation injury & $2 / 26(0.08)$ \\
\hline
\end{tabular}

CTO, chronic total occlusion; $\mathrm{PCl}$, percutaneous coronary intervention. 
example, the incidence of coronary perforation varied between $1.2 \%$ and $15.4 \%$. The conclusion of this most trusted analysis on the results of retrograde CTO PCI was that this approach has a high procedural success and an acceptable risk of procedural complications. An important publication on the temporal changes of the results is the report of a multi-center single-operator registry, which analyzed the outcome of 281 retrograde recanalizations during 7 years [17]. They divided this period into two parts and compared the results of these consecutive patient groups. The procedural success rate of the antegrade cases did not change during the entire length of the study $(89.8 \%$ vs. $91 \%)$, while there was an improving trend in the results of the retrograde procedures $(61.1 \%$ vs. $71.4 \%)$. Significantly, more calcified lesions were opened, more impaired LV functions were treated and more epicardial collaterals were used in the second period. As regards the crossing strategy, they did much less classical CART and significantly more reverse CART procedures in the second part of their study. It was stressed as an important conclusion that the systematic use of the Corsair catheter in the second period improved the results, and made the strategy more standardized with low complication rates.

\section{Complications of the retrograde approach}

There are some specific complications occurring with the retrograde strategy only. Dissection or thrombosis of the donor vessel and injury of the collaterals are complications confined to this approach. These complications might occur along with the others that are not specific to the retrograde approach like perforation or antegrade dissection of the occluded vessel, side branch occlusion, and complications of the vascular access site.

Two complications of the retrograde approach must be highlighted due to their extreme hazard. One is thrombosis of the donor vessel, particularly if this is a highly dominant artery with a large supplied area. Impairment of the blood flow in these vessels by keeping in the retrograde gear for a long time may lead to thrombotic occlusion, and hemodynamic disaster, which cannot be resuscitated in all of the cases. For this reason, the strict rule of checking activated clotting time and keeping it above $300 \mathrm{sec}$ must have an exceptional importance. Another dangerous complication can be perforation of an epicardial collateral with consequential pericardial tamponade. With availability of the contemporary guidewires and microcatheters, the use of the epicardial channels has increased. However, the exceptional risk of their inadvertent perforation must not be underestimated. Currently, the availability of embolic coils decreased this risk. But the injury and embolization of a single collateral may lead to acute myocardial infarction, as well.

With the contemporary techniques and devices, the risk of the retrograde approach is reasonably low, as has been found in the meta-analysis of El Sabbagh et al. [26] and shown in Table 3.

\section{Conclusions}

The retrograde approach in catheter-based CTO recanalization represents the most difficult challenge of coronary interventions. For a significant number of patients previously considered as cases beyond the possibilities of interventional cardiology, retrograde recanalization of CTO offers a viable option. The current diagnostic opportunities and technical developments may further facilitate planning and performing CTO procedures with a more standardized strategy and predictable outcome. There is a growing need among interventional cardiologists for widely available dedicated training programs to obtain the necessary skill for a high procedural success rate and an acceptable risk of complications in this challenging field of interventional cardiology.

\section{Acknowledgment}

The authors are grateful for the careful revision of the manuscript by Tamas Ungi.

\section{Conflict of Interest}

None declared.

\section{References}

1. Kahn, JK, and GO Hartzler. 1990. Retrograde coronary angioplasty of isolated arterial segments through saphenous vein bypass grafts. Cathet. Cardiovasc. Diagn. 20:88-93.

2. Srivatsa, SS, WD Edwards, CM Boos, et al. 1997. Histologic correlates of angiographic chronic total coronary artery occlusions. J. Am. Coll. Cardiol. 29:955-963.

3. Ozawa, N 2006. A new understanding of chronic total occlusion from a novel PCI technique that involves a retrograde approach to the right coronary artery via a septal branch and passing of the guidewire to a guiding catheter on the other side of the lesion. Catheter Cardiovasc. Interv. 68:907-913.

4. Thind, A, B Strauss, A Teitelbaum, et al. 2011. A novel method for the measurement of proximal fibrous cap puncture force in chronic total occlusions: the effect of increasing age. EuroIntervention 6:997-1002.

5. Surmely, JF, O Katoh, E Tsuchikane, et al. 2007. Coronary septal collaterals as an access for the retrograde approach 
in the percutaneous treatment of coronary chronic total occlusions. Catheter Cardiovasc. Interv. 69:826-832.

6. Brilakis, ES, JA Grantham, S Rinfret, et al. 2012. A percutaneous treatment algorithm for crossing coronary chronic total occlusions. JACC. Cardiovasc. Interv. 5: 367-379.

7. Levin, DC 1974. Pathways and functional significance of the coronary collateral circulation. Circulation 50:831-837.

8. Wu, CJ, HY Fang, CI Cheng, et al. 2011. The safety and feasibility of bilateral radial approach in chronic total occlusion percutaneous coronary intervention. Int. Heart J. 52:131-138.

9. Rinfret, S, D Joyal, CM Nguyen, et al. 2011. Retrograde recanalization of chronic total occlusions from the transradial approach; early Canadian experience. Catheter Cardiovasc. Interv. 78:366-374.

10. Werner, GS, M Ferrari, S Heinke, et al. 2003. Angiographic assessment of collateral connections in comparison with invasively determined collateral function in chronic coronary occlusions. Circulation 107:1972-1977.

11. Michael, TT, D Karmpaliotis, ES Brilakis, et al. 2013. Impact of prior coronary artery bypass graft surgery on chronic total occlusion revascularisation: insights from a multicentre US registry. Heart 99:1515-1518.

12. Brilakis, ES, D Karmpaliotis, V Patel, et al. 2012. Complications of chronic total occlusion angioplasty. Interv. Cardiol. Clin. 1:373-389.

13. Brilakis, ES 2014. Manual of coronary chronic total occlusion interventions - a step-by-step approach. Academic Press, Waltham, MA.

14. Yamane, M, M Muto, T Matsubara, et al. 2013. Contemporary retrograde approach for the recanalisation of coronary chronic total occlusion: on behalf of the Japanese Retrograde Summit Group. EuroIntervention 9:102-109.

15. Lee, NH, HS Seo, JH Choi, et al. 2010. Recanalization strategy of retrograde angioplasty in patients with coronary chronic total occlusion - analysis of 24 cases, focusing on technical aspects and complications. Int. J. Cardiol. 144:219-229.

16. Kimura, M, O Katoh, E Tsuchikane, et al. 2009. The efficacy of a bilateral approach for treating lesions with chronic total occlusions. The CART (Controlled Antegrade and Retrograde subintimal Tracking) Registry. JACC. Cardiovasc Interv. 2:1135-1141.

17. Muramatsu, T, R Tsukahara, Y Ito, et al. 2013. Changing strategies of the retrograde approach for chronic total occlusion during the past 7 years. Catheter Cardiovasc. Interv. 81:E178-E185.

18. Stojkovic, S, G Sianos, O Katoh, et al. 2012. Efficiency, safety, and long-term follow-up of retrograde approach for cto recanalization: initial (Belgrade) experience with international proctorship. J. Interv. Cardiol. 25:540-548.

19. Galassi, AR, SD Tomasello, N. Reifart, et al. 2011. Inhospital outcomes of percutaneous coronary intervention in patients with chronic total occlusion: insights from the ERCTO (European Registry of Chronic Total Occlusion) registry. EuroIntervention 7:472-479.

20. Karmpaliotis, D, TT Michael, ES Brilakis, et al. 2012. Retrograde coronary chronic total occlusion revascularization procedural and in-hospital outcomes from a multicenter registry in the United States. JACC. Cardiovasc. Interv. 5:1273-1279.

21. Surmely, JF, E Tsuchikane, O Katoh, et al. 2006. New concept for CTO recanalization using controlled antegrade and retrograde subintimal tracking: the CART technique. J. Invasive Cardiol. 18:334-338.

22. Mozid, AM, JR Davies, and JC Spratt. 2014. The utility of a guideliner ${ }^{\mathrm{TM}}$ catheter in retrograde percutaneous coronary intervention of a chronic total occlusion with reverse cart-the "capture" technique. Catheter Cardiovasc. Interv. 83:929-932.

23. Joyal, D, CA Thompson, JA Grantham, et al. 2012. The retrograde technique for recanalization of chronic total occlusions. A step-by-step approach. JACC. Cardiovasc. Interv. 5:1-11.

24. Biondi-Zoccai, GL, M Bollati, C Moretti, et al. 2008. Retrograde percutaneous recanalization of coronary chronic total occlusions: outcomes from 17 patients. Int. J. Cardiol. 130:118-120.

25. Rathore, S, O Katoh, H Matsuo, et al. 2009. Retrograde percutaneous recanalization of chronic total occlusion of the coronary arteries: procedural outcomes and predictors of success in contemporary practice. Circ. Cardiovasc. Interv. 2:124-132.

26. El Sabbagh, A, VG Patel, OM Jeroudi, et al. 2014. Angiographic success and procedural complications in patients undergoing retrograde percutaneous coronary chronic total occlusion interventions: a weighted metaanalysis of 3482 patients from 26 studies. Int. J. Cardiol. 174:243-248. 